\section{POS1041 PREVALENCE, INCIDENCE AND ANTIRHEUMATIC DRUG USE IN PSORIATIC ARTHRITIS (PSA) IN NORWAY}

A. Kerola ${ }^{1,2}$, J. Sexton ${ }^{1}$, S. Rollefstad ${ }^{1}$, G. Wibetoe ${ }^{1}$, C. S. Crowson ${ }^{3}$, E. Haavardsholm ${ }^{1}$, T. K. Kvien ${ }^{1}$, A. G. Semb ${ }^{1}$. ${ }^{1}$ Diakonhjemmet Hospital, Division of Rheumatology and Research, Oslo, Norway; ${ }^{2}$ Päijät-Häme Joint Authority for Health and Wellbeing, Rheumatology, Lahti, Finland; ${ }^{3}$ Mayo Clinic, Department of Quantitative Health Sciences, Rochester, Minnesota, United States of America

Background: Incidence estimates of PsA in Norway have varied from $6.9 / 100,000$ person-years (pyrs) in Northern Norway to $41.3 / 100,000$ pyrs in Central Norway, and point prevalence estimates have ranged from 1.3 to 6.9 per 1,000 adult inhabitants ${ }^{1,2}$, while nationwide epidemiologic data on PsA in Norway have been lacking.

Objectives: To estimate prevalence, incidence and use of disease-modifying antirheumatic drugs (DMARDs) among PsA patients in Norway.

Methods: The Norwegian Cardio-Rheuma register includes pseudonymized data from the total Norwegian population $\geq 18$ years of age during 2008-2017, identified from the National Population register. Demographic and socioeconomic data were retrieved from Statistics Norway. Data on public or private somatic specialized care episodes were collected from the Norwegian Patient register (NPR) [ICD-10 codes for diagnoses and medical procedure codes for biologic DMARD infusions]. Information on dispensed DMARD prescriptions was captured from the Norwegian Prescription Database. Based on NPR data, PsA cases were defined as persons fulfilling three criteria: 1) 1st episode with ICD-10 code M07.0-M07.3 or L40.5 as main or contributory diagnosis (index date), 2) 2nd episode with code M07.0-M07.3 or L40.5 within 2-year period following index date, 3) an episode in internal medicine or rheumatology clinic with recorded M07.0-M07.3 or L40.5 within 2 years from index date. Years 2008-2010 served as a look-back period to identify prevalent PsA cases. To estimate pyrs at risk, we calculated number of individuals aged $\geq 18$ years living in Norway on the $1^{\text {st }}$ of January of each year 2011-2015 multiplied by one year (prevalent PsA cases excluded). Age- and sex-standardized incidence rates were calculated with 5 -year age groups using the Norwegian adult population on January $1^{\text {st }} 2015$ as the standard.

Results: During the look-back period 2008-2010, 7,697 cases fulfilled the PsA definition. In total, 6,183 incident PsA cases were identified during 2011-2015 (incidence 32/100,000 pyrs, 28 among men and 35 among women). Based on a sensitivity analysis comprising 5,065 PsA cases with no dispensed DMARD prescriptions $\geq 12$ months before index date, incidence was slightly lower (26/100,000 pyrs). Patient characteristics and DMARD use are shown in Table 1. The incidence was highest among those aged 50-59 years in both sexes (Figure 1). PsA incidence was lower among those with higher education level (crude/ age- and sex-standardized incidence per 100,000 pyrs for those below upper secondary education $34 / 38$, upper secondary or post-secondary non-tertiary education 36/36, higher education 26/25). Point prevalence of PsA was 3.3/1,000 adult inhabitants on January $1^{\text {st }} 2016$.

Table 1. Characteristics and treatment penetration of incident PsA patients 2011-2015

\begin{tabular}{|c|c|c|c|c|}
\hline & \multicolumn{2}{|l|}{ All } & \multicolumn{2}{|c|}{$\begin{array}{l}\text { Excluding cases with } \\
\text { DMARDs }>1 \text { yr prior to } \\
\text { index date }\end{array}$} \\
\hline $\mathbf{N}$ & 6183 & & 5065 & \\
\hline Women, $\mathrm{n}(\%)$ & $3442(55.7)$ & & $2783(54.9)$ & \\
\hline Age at index date, median (IQR) & $50.5(40.7-$ & 59.8) & $49.9(40.2-$ & 59.3) \\
\hline Use of DMARDs after index date, $n(\%)$ & 12 months & 24 months & 12 months & 24 months \\
\hline Any conventional DMARD & $3706(59.9)$ & $4048(65.4)$ & $2894(57.1)$ & $3184(62.9)$ \\
\hline Methotrexate & $3313(53.6)$ & $3650(59.0)$ & $2638(52.1)$ & $2933(57.9)$ \\
\hline Sulfasalazine & $440(7.1)$ & $586(9.5)$ & $330(6.5)$ & $457(9.0)$ \\
\hline Any biologic DMARD & $842(13.6)$ & $1197(19.4)$ & $485(9.6)$ & $771(15.2)$ \\
\hline TNF-inhibitors & $810(13.1)$ & $1154(18.7)$ & $477(9.4)$ & $758(15.0)$ \\
\hline Oral glucocorticoids & $1773(28.7)$ & $2240(36.2)$ & $1449(28.6)$ & $1807(35.7)$ \\
\hline Any DMARD or glucocorticoids & $4365(70.6)$ & $4742(76.7)$ & 3384 & 3725 \\
\hline
\end{tabular}

Conclusion: Our estimate of PsA incidence and prevalence are in the mid-range compared to studies from smaller regions in Norway. Methotrexate was initiated for more than half of PsA cases within one year from index date, whereas 19\% had used biologic DMARDs within two years.

REFERENCES:

[1] Hoff M, Gulati A, Romundstad P et al. Prevalence and incidence rates of psoriatic arthritis in central Norway: data from the Nord-Trondelag health study. Ann Rheum Dis 2015;74:60-64.

[2] Nossent J \& Gran J. Epidemiological and clinical characteristics of psoriatic arthritis in northern Norway. Scand J Rheumatol 2009; 8:251-5.

Acknowledgements: This work has been supported by a research grant from FOREUM Foundation for Research in Rheumatology.

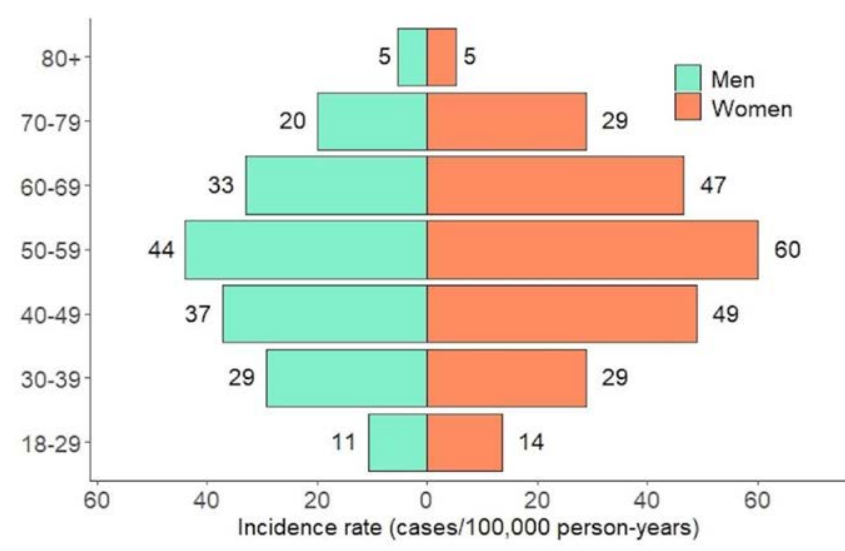

Figure 1.

Disclosure of Interests: Anne Kerola Speakers bureau: Boehringer-Ingelheim, Consultant of: Pfizer, Gilead and Boehringer-Ingelheim, Joseph Sexton: None declared, Silvia Rollefstad: None declared, Grunde Wibetoe: None declared, Cynthia S. Crowson: None declared, Espen Haavardsholm: None declared, Tore K. Kvien Speakers bureau: Amgen, Celltrion, Egis, Evapharma, Ewopharma, Hikma, Oktal, Sandoz, Sanofi, Consultant of: AbbVie, Amgen, Biogen, Celltrion, Eli Lilly, Gilead, Mylan, Novartis, Pfizer, Sandoz, Sanofi, Grant/research support from: research funding to Diakonhjemmet Hospital from AbbVie, Amgen, BMS, MSD, Pfizer and UCB, Anne Grete Semb Speakers bureau: AbbVie, Bayer, Lilly, Novartis, Sanofi, Consultant of: Sanofi, Grant/research support from: Collaborative research support from Lilly, outside the submitted work. DOI: 10.1136/annrheumdis-2021-eular.980

\section{POS1042 EFFICACY AND SAFETY OF DEUCRAVACITINIB, AN ORAL, SELECTIVE TYROSINE KINASE 2 (TYK2) INHIBITOR, COMPARED WITH PLACEBO AND APREMILAST IN MODERATE TO SEVERE PLAQUE PSORIASIS: RESULTS FROM THE PHASE 3 POETYK PSO-1 STUDY}

A. Armstrong ${ }^{1}$, M. Gooderham ${ }^{2}$, R. B. Warren ${ }^{3}$, K. Papp ${ }^{4}$, B. Strober ${ }^{5,6}$, D. Thaçi ${ }^{7}$, E. Colston ${ }^{8}$, J. Throup ${ }^{9}$, S. Kundu ${ }^{10}$, S. Banerjee ${ }^{11}$, A. Blauvelt ${ }^{12}$ ${ }^{1}$ Keck School of Medicine, University of Southern California, Los Angeles, United States of America; ${ }^{2}$ SKiN Center for Dermatology, Queen's University and Probity Medical Research, Peterborough, Canada; ${ }^{3}$ Dermatology Center, Salford Royal NHS Foundation Trust Hospital, Manchester NIHR Biomedical Research Center, University of Manchester, Manchester, United Kingdom; ${ }^{4}$ Clinical Research and Probity Medical Research, K. Papp Clinical Research, Waterloo, Canada; ${ }^{5}$ Yale University, Dermatology, New Haven, United States of America; ${ }^{6}$ Central Connecticut Dermatology Research, Co-founder, Cromwell, United States of America; ${ }^{7}$ University of Lübeck, Comprehensive Centre for Inflammation Medicine, Lübeck, Germany; ${ }^{8}$ Bristol Myers Squibb, Dermatology, Princeton, United States of America; ${ }^{9}$ Bristol Myers Squibb, Immunoscience $R \& D$, Princeton, United States of America; ${ }^{10}$ Bristol Myers Squibb, Global Biometrics Sciences, Princeton, United States of America: ${ }^{11}$ Bristol Myers Squibb, Rheumatology and Dermatology, Princeton, United States of America; ${ }^{12}$ Oregon Medical Research Center, Dermatology, Portland, United States of America

Background: Tyrosine kinase 2 (TYK2) is an intracellular kinase that mediates interleukin (IL)-23, IL-12, and interferon (IFN) $\alpha / \beta$ signaling. Deucravacitinib is a novel, oral, selective inhibitor of TYK2 acting via binding to the TYK2 regulatory domain. ${ }^{1}$ Phase 2 results showed deucravacitinib was efficacious and well tolerated versus placebo in patients with moderate to severe plaque psoriasis or active psoriatic arthritis. ${ }^{2,3}$ No herpes zoster infections, opportunistic infections, thromboembolic events, or hematologic or lipid abnormalities characteristic of Janus kinase (JAK) 1-3 inhibitors were reported in the Phase 2 trials. ${ }^{2,3}$

Objectives: To compare the efficacy and safety of deucravacitinib versus placebo and apremilast in plaque psoriasis.

Methods: This Phase 3, double-blinded, 52-week study (NCT03624127) randomized patients with moderate to severe plaque psoriasis (BSA $\geq 10 \%$, PASI $\geq 12$, sPGA $\geq 3$ ) to deucravacitinib $6 \mathrm{mg}$ once daily, placebo, or apremilast $30 \mathrm{mg}$ twice daily (2:1:1). Patients receiving placebo were switched to deucravacitinib at Week 16; apremilast-treated patients not achieving PASI 50 at Week 24 were switched to deucravacitinib. Coprimary endpoints were PASI 75 and SPGA 0/1 response versus placebo at Week 16 . Key secondary endpoints included superiority versus apremilast, assessed via multiple measures.

Results: 666 patients were randomized. Demographic and baseline disease characteristics were balanced across groups; mean age was 46.1 years, mean 
disease duration was 17.3 years, $18.2 \%$ of patients had psoriatic arthritis at baseline, and $38.9 \%$ had previously used biologic therapy. Mean BSA involvement at baseline was $26.3 \%$, mean PASI was 21.4 , and the percentage with severe SPGA (score $=4$ ) at baseline was $21.2 \%$. Significantly greater proportions of patients in the deucravacitinib versus placebo and apremilast arms achieved PASI 75 (58.7\% vs $12.7 \%$ vs $35.1 \%$, respectively; $P<0.0001)$ and sPGA $0 / 1(53.6 \%$ vs $7.2 \%$ vs $32.1 \%$, respectively; $P<0.0001$ ) response at Week 16 (Figure 1). Deucravacitinib was also superior to apremilast at Week 24 , with $69.0 \%$ versus $38.1 \%$ of patients achieving PASI 75 and $58.4 \%$ versus $31.0 \%$ achieving SPGA $0 / 1$ ( $P<0.0001$ for both). In addition, DLQI 0/1 responses at Week 16 were significantly higher with deucravacitinib versus placebo and apremilast, demonstrating improved quality of life $(40.7 \%$ vs $10.6 \%$ vs $28.6 \%$, respectively; Figure 1$)$. During the 16-week, placebo-controlled period, the most common AEs ( $\geq 5 \%$ in any arm) were nasopharyngitis, upper respiratory tract infection, headache, diarrhea, and nausea (Table 1). Frequencies of SAEs and treatment discontinuations due to AEs were low (Table 1).

Table 1. Summary of adverse events (AEs) through Week 16

\begin{tabular}{llll}
\hline Patients, $\mathbf{n}(\%)$ & Deucravacitinib & Placebo & Apremilast \\
& $\mathbf{n = 3 3 2}$ & $\mathbf{n = 1 6 5}$ & $\mathbf{n = 1 6 8}$ \\
\hline Any AEs & $176(53.0)$ & $70(42.4)$ & $93(55.4)$ \\
Severe AEs & $5(1.5)$ & $7(4.2)$ & $5(3.0)$ \\
Serious AEs & $7(2.1)$ & $9(5.5)$ & $4(2.4)$ \\
AEs leading to treatment discontinuation & $6(1.8)$ & $7(4.2)$ & $10(6.0)$ \\
Most common AEs $(\geq \mathbf{5 \%}$ in any arm) & $21(6.3)$ & $7(4.2)$ & $14(8.3)$ \\
Nasopharyngitis & $21(6.3)$ & $6(3.6)$ & $3(1.8)$ \\
Upper respiratory tract infection & $16(4.8)$ & $5(3.0)$ & $17(10.1)$ \\
Headache & $13(3.9)$ & $6(3.6)$ & $17(10.1)$ \\
Diarrhea & $7(2.1)$ & $4(2.4)$ & $19(11.3)$ \\
Nausea & & & \\
\hline
\end{tabular}

Conclusion: Deucravacitinib demonstrated superiority versus placebo and apremilast across multiple efficacy endpoints in patients with moderate to severe plaque psoriasis, and was generally well tolerated. Overall, the efficacy and safety profile of deucravacitinib was consistent with that observed in the Phase 2 plaque psoriasis and psoriatic arthritis trials. ${ }^{2,3}$

REFERENCES:

[1] Burke JR et al. Sci Trans/ Med. 2019;11:1-16.

[2] Papp K et al. N Engl J Med. 2018;379:1313-21.

[3] Mease PJ et al. Presented at: Annual Scientific Meeting of the American College of Rheumatology; November 5-9, 2020; Virtual meeting.

Figure. Efficacy responses at Week 16

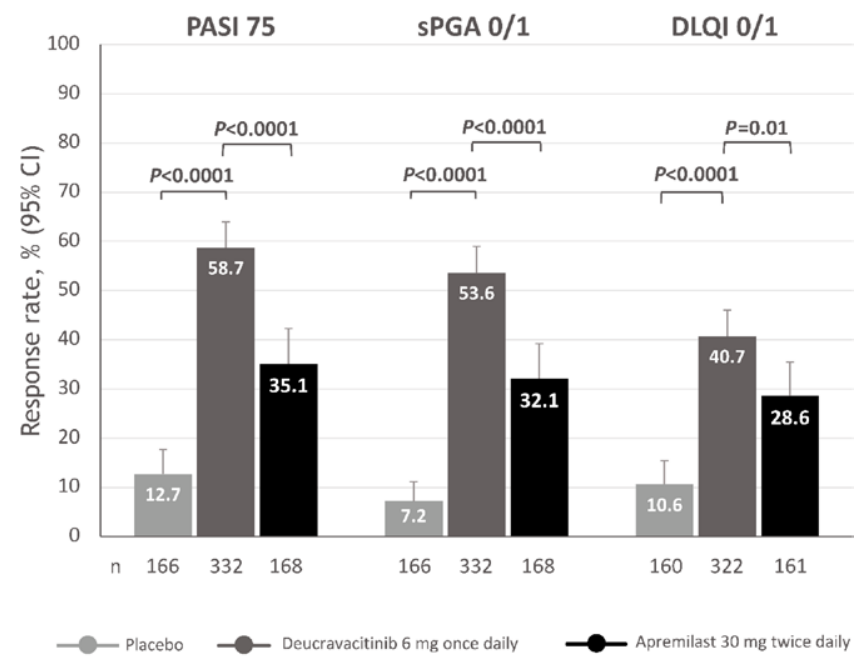

Acknowledgements: This study was sponsored by Bristol Myers Squibb. Professional medical writing assistance was provided by Peloton Advantage, LLC, an OPEN Health company, and funded by Bristol Myers Squibb.

Disclosure of Interests: April Armstrong Consultant of: Grants and personal fees from AbbVie, Bristol Myers Squibb, Eli Lilly, Janssen, Leo Pharma, Novartis; Personal fees from Boehringer Ingelheim/Parexel, Celgene, Dermavant, Genentech,
GlaxoSmithKline, Menlo Therapeutics, Merck, Modernizing Medicine, Ortho Dermatologics, Pfizer, Regeneron, Sanofi Genzyme, Science 37, Sun Pharma, Valeant, Grant/research support from: Grants: Dermira, Kyowa Hakko Kirin, and UCB, outside the submitted work; Grants and personal fees from AbbVie, Bristol Myers Squibb, Eli Lilly, Janssen, Leo Pharma, Novartis, Melinda Gooderham Shareholder of: Speakers bureau, consultant, investigator/advisor: AbbVie, Akros, Amgen, Arcutis, Boehringer Ingelheim, Bristol Myers Squibb, Celgene, Dermira, Eli Lilly, Galderma, GlaxoSmithKline, Incyte, Janssen, Kyowa Hakko Kirin, Leo Pharma, Merck, Medlmmune, Novartis, Pfizer, Regeneron, Roche, Sanofi Genzyme, Takeda, UCB, Valeant, Richard B. Warren Consultant of: AbbVie, Almirall, Amgen, Boehringer Ingelheim, Celgene, Janssen, Leo Pharma, Eli Lilly, Novartis, Pfizer, Sanofi, Xenoport, UCB, Grant/research support from: AbbVie, Almirall Amgen, Celgene, Janssen, Eli Lilly, Leo Pharma, Novartis, Pfizer, UCB, Kim Papp Speakers bureau: AbbVie, Amgen, Astellas, Celgene, Eli Lilly, Galderma, Janssen, Kyowa Hakko Kirin, Leo Pharma, Merck Sharp \& Dohme, Novartis, Pfizer, Valeant, Consultant of: Scientific officer/steering committee/advisory board: AbbVie, Akros, Amgen, Anacor, Astellas, Baxter, Boehringer Ingelheim, Bristol Myers Squibb, Celgene, Dow Pharma, Eli Lilly, Galderma, Janssen, Kyowa Hakko Kirin, Merck Sharp \& Dohme, Merck Serono, Novartis, Pfizer, Regeneron, Sanofi-Aventis/Genzyme, Valeant, Grant/research support from: AbbVie, Akros, Allergan, Amgen, Anacor, Arcutis, AstraZeneca, Baxalta, Boehringer Ingelheim, Bristol Myers Squibb, Celgene, Coherus, Dermira, Dow Pharma, Eli Lilly, Galderma, Genentech, GlaxoSmithKline, Janssen, Kyowa Hakko Kirin, Leo Pharma, Medlmmune, Meiji Seika Pharma, Merck Serono, Novartis, Pfizer, Regeneron, Roche, Sanofi-Aventis/Genzyme, Takeda, UCB, Valeant;

Consultant: AbbVie, Akros, Amgen, Arcutis, Astellas, AstraZeneca, Baxalta, Baxter, Boehringer Ingelheim, Bristol Myers Squibb, CanFite, Celgene, Coherus, Dermira Dow Pharma, Eli Lilly, Forward Pharma, Galderma, Genentech, Janssen, Kyowa Hakko Kirin, Leo Pharma, Meiji Seika Pharma, Merck Sharp \& Dohme, Merck Serono, Mitsubishi Pharma, Novartis, Pfizer, Regeneron, Roche, Sanofi-Aventis/ Genzyme, Takeda, UCB, Valeant; Honoraria: AbbVie, Akros, Amgen, Baxter, Boehringer Ingelheim, Celgene, Coherus, Eli Lilly, Forward Pharma, Galderma GlaxoSmithKline, Janssen, Kyowa Hakko Kirin, Merck Sharp \& Dohme, Merck Serono, Novartis, Pfizer, Takeda, UCB, Valeant, Bruce Strober Speakers bureau: AbbVie, Janssen, Eli Lilly, Ortho Dermatologics, Consultant of: Honoraria or consultation fees: AbbVie, Almirall, Amgen, Arena, Boehringer Ingelheim, Bristol Myers Squibb, Celgene, Dermavant, Dermira, Eli Lilly, GSK, Janssen, Kyowa Hakko Kirin, Leo Pharma, Medac, Meiji Seika Pharma, Novartis, Ortho Dermatologics, Pfizer, Regeneron, Sanofi-Genzyme, Sun Pharma, UCB; Scientific Director (consulting fee): Corrona Psoriasis Registry; Investigator: AbbVie, Corrona Psoriasis Registry, Dermavant, Dermira., Diamant Thaçi Paid instructor for: Lectures: AbbVie, Almirall, Amgen, DS-Pharma, Janssen, Leo Pharma, MSD, Novartis, Pfizer, RochePosay, Sandoz-Hexal, Sanofi, Target-Solution, UCB; Scientific advisory board: AbbVie, Amgen, Celgene, DS Pharma, Eli Lilly, Galapagos, Janssen-Cilag, Leo Pharma, Morphosis, MSD Novartis, Pfizer, Sandoz, Sanofi, UCB., Consultant of: Consultant: AbbVie, Almirall, Celgene, Dignity, Galapagos, Leo Pharma, Maruho, Mitsubishi, Novartis, Pfizer, Xenoport, Grant/research support from: Research support/principal investigator (clinical trials): AbbVie, Almirall, Amgen, Biogen Idec, Boehringer Ingelheim, Celgene, Chugai, Dermira, DS-Pharma, Eli Lilly, Galderma, GSK, Janssen-Cilag, Leo, MSD, Novartis, Pfizer, Regeneron, Roche Sandoz-Hexal, Sanofi, UCB, Elizabeth Colston Shareholder of: Bristol Myers Squibb, Employee of: Bristol Myers Squibb, John Throup Shareholder of: Bristo Myers Squibb, Employee of: Bristol Myers Squibb, Sudeep Kundu Shareholder of: Bristol Myers Squibb, Employee of: Bristol Myers Squibb, Subhashis Banerjee Shareholder of: Bristol Myers Squibb, Employee of: Bristol Myers Squibb, Andrew Blauvelt Consultant of: Scientific adviser and/or clinical study investigator for AbbVie, Aligos, Almirall, Arena, Athenex, Boehringer Ingelheim, Bristol Myers Squibb, Dermavant, Eli Lilly, Evommune, Forte, Galderma, Incyte, Janssen, Leo Pharma Novartis, Pfizer, Rapt, Regeneron, Sanofi Genzyme, Sun Pharma, UCB Pharma. DOI: 10.1136/annrheumdis-2021-eular.1002

\section{\begin{tabular}{|l|l|l}
\hline POS1043 NETAKIMAB REDUCES PSORIATIC ARTHRITIS \\
\hline
\end{tabular} ACTIVITY IN PATIENTS WITH OR WITHOUT AXIAL DISEASE: SUBANALYSIS OF THE PATERA STUDY}

T. Korotaeva ${ }^{1}$, I. Gaydukova ${ }^{2}$, V. Mazurov ${ }^{2}$, A. Samtsov ${ }^{3}$, V. Khayrutdinov ${ }^{3}$, A. Bakulev ${ }^{4}$, A. Kundzer ${ }^{5}$, N. Soroka ${ }^{6}$, A. Eremeeva ${ }^{7} .{ }^{1}$ Nasonova Research Institute of Rheumatology, Laboratory of Spondyloarthritides and Psoriatic Arthritis, Moscow, Russian Federation; ${ }^{2}$ Mechnikov North-Western State Medical University, Department of Therapy and Rheumatology of Temporary Disability and Medical Care Quality Expertise, St-Petersburg, Russian Federation; ${ }^{3}$ Kirov Military Medical Academy, Department of Skin and Veneral Diseases, St-Petersburg, Russian Federation; ${ }^{4}$ Razumovsky Saratov State Medical University, Department of Dermatovenereology and Cosmetology, Saratov, Russian Federation; ${ }^{5}$ Belarusian Medical Academy of Postgraduate Education, Department of Cardiology and Rheumatology, Minsk, Belarus; ${ }^{6}$ Belarusian State Medical University, Department of Internal Diseases 